\title{
Le conflit ukrainien, des enjeux géopolitiques et géoéconomiques
}

\author{
Pascal Marchand
}

\section{Q OpenEdition}

Journals

Édition électronique

URL : http://journals.openedition.org/echogeo/13976

DOI : 10.4000/echogeo. 13976

ISSN : 1963-1197

\section{Éditeur}

Pôle de recherche pour l'organisation et la diffusion de l'information géographique (CNRS UMR 8586)

\section{Référence électronique}

Pascal Marchand, «Le conflit ukrainien, des enjeux géopolitiques et géoéconomiques », EchoGéo [En ligne], Sur le Vif, mis en ligne le 31 octobre 2014, consulté le 10 décembre 2020. URL : http:// journals.openedition.org/echogeo/13976; DOI : https://doi.org/10.4000/echogeo.13976

Ce document a été généré automatiquement le 10 décembre 2020.

\section{(c) (i) (9)}

EchoGéo est mis à disposition selon les termes de la licence Creative Commons Attribution - Pas d'Utilisation Commerciale - Pas de Modification 4.0 International 


\title{
Le conflit ukrainien, des enjeux géopolitiques et géoéconomiques
}

\author{
Pascal Marchand
}

1 A propos de l'embrasement de l'Ukraine à partir de novembre 2013, l'ancien ambassadeur de France à Kiev, Philippe de Suremain, expliquait le 5 juin à Lyon à l'Institut des Hautes Études de Défense Nationale (IHEDN) : «on n'avait rien vu venir ». Confirmant plusieurs informations de presse, Aurélie Allain (2014) écrit qu'aucune chancellerie «n'a vu venir » l'opération russe en Crimée.

2 Cet accès de cécité montre l'utilité d'une approche géopolitique. Celle qui sera développée ici repose sur l'association des données géoéconomiques et stratégiques. Elle permet de comprendre les "intérêts objectifs » d'un acteur étatique et d'envisager l'éventail des possibles ce qui permet d'éviter de voir se produire des évènements qu'on «n'avait pas vu venir ». D'autres facteurs importants, d'ordre religieux, culturel ou historique, peuvent certes entrer en compte, c'est le cas en Ukraine. Ils ne seront qu'évoqués ici, le lecteur pouvant se reporter à la bibliographie indiquée.

\section{L'Ukraine, un pays fragile}

\section{Une création récente}

3 Avant 1991, l'Ukraine n'avait jamais existé en tant qu'État, sujet reconnu de droit international. Sur l'isthme Baltique - mer Noire, il n'y avait pas eu d'État avant la formation de la Rous en 856. Elle comprenait seulement l'ouest de l'Ukraine actuelle et la région de Kiev à l'exclusion du sud et de l'est. Elle s'étendait principalement sur les territoires actuels de la Biélorussie et de la Russie. Abattue par les Tatars en 1240, elle se fragmenta en principautés. Dans l'empire constitué par Moscou sur cet espace, la place de l'Ukraine actuelle était occupée par plusieurs provinces, dont aucune ne portait ce nom.

4 Lors de l'éclatement de l'empire en 1917, deux gouvernements locaux, non reconnus, se sont formés sur le territoire de l'actuelle Ukraine: l'un, nationaliste, à Kiev, l'autre, 
bolchévik, à Kharkov. Ce dernier a pris Kiev en février 1918 puis les forces allemandes l'en ont chassé en mars, y installant un gouvernement à leur solde. La république d'Ukraine occidentale fondée à Lviv en Galicie par les nationalistes en novembre 1918 a repris le contrôle de Kiev en janvier 1919, mais l'armée polonaise avait entrepris dès novembre 1918 la conquête de la Galicie, dont elle reste maîtresse en juin 1919. Elle prend Kiev en mai 1920 et combat le pouvoir de Kharkov, devenu République Soviétique d'Ukraine depuis mars 1919, qui la repousse. Par la paix de 1921, la Pologne garde la Galicie et la Volhynie, fiefs des nationalistes. Les bolchéviks constituent une RSS d'Ukraine, simple subdivision administrative de l'URSS.

5 L'Ukraine indépendante de 1991 est cette République Socialiste Soviétique d'Ukraine, restructurée par Staline en 1945. Elle regroupe des régions qui depuis des siècles ont connu des histoires différentes :

- trois régions sont issues de la Rous du Xe-XIIe siècle, dont la capitale fut successivement Novgorod (856), Kiev (882), Vladimir (1169) ${ }^{1}$ : le Dniepr moyen et Kiev, berceau de la christianisation de la Rous, rallié à Moscou après sa révolte contre l'occupant catholique polonais, en 1654 ; l'Ukraine du centre-ouest et la Volhynie, davantage polonisées, intégrées à l'empire russe depuis 1793 ; laGalicie, encore plus occidentale, qui n'avait jamais été dirigée par Moscou avant 1945, puisque sous contrôle polonais depuis 1347, autrichien de 1772 à 1919, puis de nouveau polonais de 1921 à 1939 ;

- deux régions n'ont jamais fait partie de la Rous du Xe-XIIe siècle et ont toujours été dirigées par un pouvoir cis à Moscou avant 1991, jamais à Kiev : l'Ukraine-Sloboda, à l'est, autour de Kharkov, ville fondée en 1503 ; la Nouvelle Russie, au sud (provinces russes d'Ekaterinoslav et de Kherson), conquise seulement par la Russie de Catherine II à la fin du XVIIIe siècle et peuplée de colons russes, ukrainiens et allemands au XIXe siècle ;

- enfin trois régions sont annexées par Staline en 1945 : la Ruthénie subcarpatique, qui en 1919 avait préféré se rallier à la Tchécoslovaquie plutôt que de passer sous le contrôle du gouvernement ukrainien de Galicie; la Bucovine (oblast de Tchernovtsy), partie septentrionale de la Moldavie historique, intégrée à l'Autriche au XVIIIe siècle, puis à la Roumanie, de 1918 à 1945 ; le Budjak, façade maritime de la Bessarabie².

6 La Crimée, conquise par Catherine II, qui constituait la province russe de Tauride (cartes dans Channon, 1997), lui a été rattachée par décision de Khrouchtchev (d'origine ukrainienne), en 1954.

7 La RSS d'Ukraine a en revanche été amputée de la Transnistrie (Ruze, 1999). Cette zone, peuplée d'Ukrainiens et de Russes, a été rattachée en 1945 à la RSS de Moldavie, alors qu'elle ne faisait pourtant pas partie de la Bessarabie (Sellier, 1991).

8 L'unité de régions historiquement aussi diverses ne va pas de soi. Surtout que leur situation géographique en font depuis cinq siècles un enjeu entre deux messianismes inspirés par deux Églises chrétiennes, ceux de Varsovie et de Moscou, pour ne rien dire des influences occasionnelles de Berlin, Vienne et Bucarest. En 1991, le nouvel État était donc en quête d'éléments d'identité partagée mais dès ce moment, d'anciens clivages ont rejoué.

\section{Un défaut de facteurs d'unité}

9 Sur le plan religieux, le pays fut le théâtre pendant la décennie quatre-vingt-dix d'une « guerre des églises » entre fidèles de trois obédiences d'une même Église orthodoxe, pour le contrôle des lieux de culte. 
10 Depuis la christianisation de 988, le métropolite de la Rous orthodoxe résidait à Kiev, cependant l'insécurité l'avait contraint au XIII ${ }^{\mathrm{e}}$ siècle à gagner le nord-est de la Rous, la région de Vladimir, plus sûre. En 1326, le prince de Moscou lui a offert un domaine près de sa ville qui devint ainsi le centre de l'Église orthodoxe "de toutes les Russies». Historiquement, le Patriarche de Moscou est l'héritier légitime du métropolite de Kiev.

11 Mais lors de l'épisode de 1918-19, le gouvernement nationaliste ukrainien a créé une Église autocéphale ukrainienne, reconnue par Constantinople. Avec la victoire des Bolchéviks, elle a trouvé refuge dans l'importante diaspora ukrainienne du Canada. En 1991, le nouveau gouvernement ukrainien a créé lui aussi une Église orthodoxe autocéphale ukrainienne, cette fois non reconnue par Constantinople. L'Église exilée au Canada étant revenue, en Ukraine, l'orthodoxie est divisée en trois obédiences, celle du patriarcat de Moscou et deux Eglises autocéphales (Thual, 1993 ; Boyko, 2004). En 2007, 11233 paroisses dépendaient du Patriarcat historique, celui de Moscou, $3963 \mathrm{du}$ Patriarcat de Kiev, 1178 de l'Église autocéphale ukrainienne de 1918 (Galtsyan, 2012). Il existe par ailleurs une Église uniate, ou gréco-catholique, de rite orthodoxe, qui reconnaît la suprématie du pape de Rome. Créée par les Polonais au XVII siècle pour « rallier les hérétiques à la Sainte Croix» (Slekowa, 1988), elle n'est implantée qu'à l'Ouest de l'Ukraine.

12 Les Églises orthodoxes étant autocéphales, c'est-à-dire organisées par l'État, elles légitiment d'une certaine façon ce niveau de pouvoir politique. Pour un État qui apparaît par scission avec une entité politique plus vaste, l'existence d'une entité religieuse propre est un élément important d'affirmation politique. Mais des fidèles peuvent voir cette partition comme une trahison de la communauté spirituelle. L'enjeu politique de ce conflit, à priori religieux, est très fort.

13 Sur le plan linguistique, l'unité n'est pas plus assurée. La définition de l'ukrainien officiel a fait gravement problème dans la décennie quatre-vingt-dix en raison de violentes divergences au sein du milieu se réclamant de l'ukraïnité (Besters-Dilger, 2005).

Dans la partie de l'ex Rous sous contrôle polonais du XIV ${ }^{e}$ au XVIII ${ }^{e}$ siècle, la Pologne avait favorisé l'usage du polonais. Le « ruthène », langue des paysans, était méprisé. Dans ce qu'on appelait la Malo Polska (Petite Pologne), les populations ruthènes étaient considérées comme ne se différenciant que par leur dialecte du tronc principal de la nation polonaise (Kalembka, 1988). En 1845, le prince Czartoryski déclarait « Ruthènes et Lituaniens forment avec nous une Nation ».

15 Fin XVIII ${ }^{e}$, hormis la Galicie, acquise par l'Autriche, la Malo Polska est passée sous le contrôle de Moscou. Pour les autorités russes, ses habitants ne sont que des «PetitRussiens », utilisant « un dialecte qui est du russe, simplement corrompu par l'influence de la Pologne » (Kappeler, 1994). L'enseignement de l'ukrainien y était interdit.

16 En revanche, en Galicie, l'empereur d'Autriche s'est posé en père protecteur des « Ruthènes ». L'enseignement primaire était dispensé en ukrainien. Des journaux furent autorisés en ukrainien à partir de 1848. Ceci imposait le choix, parmi ses nombreuses inflexions, des canons d'une langue ukrainienne standard. Une chaire de philologie ukrainienne fut donc créée en 1849 à Lemberg (Besters-Dilger, 2005). Les bases de la langue ukrainienne ont ainsi été jetées dans l'Empire d'Autriche. L'URSS développa elle aussi à partir des années vingt l'usage de l'ukrainien, mais en choisissant une variante de l'Ukraine centrale, plus proche du russe que celui qui avait été codifié en Galicie. 

2010 n'est toujours pas publié). Comme dans presque toute l'Europe centrale et orientale, il distingue la "citoyenneté » (ukrainienne) de la «nationalité», qui correspond au groupe ethnique auquel l'individu se rattache: $77,8 \%$ de la population se déclare de nationalité ukrainienne, $17,3 \%$ de nationalité russe, et $4,9 \%$ d'une autre nationalité (Rowland, 2004). entre Russie et Ukraine, $29,6 \%$ des habitants du pays se déclarent de langue maternelle russe. La langue ukrainienne n'est donc la langue maternelle que de $68,5 \%$ de la population : un nombre important de personnes se déclarant de nationalité ukrainienne se considère de langue maternelle russe (All-Ukrainian population census, 2001).

L'ukrainien est plus répandu dans les campagnes, mais sous un grand nombre de formes dialectales. Presque toute la population comprend le russe et passe d'une langue à l'autre en fonction de l'interlocuteur, mais la majorité parle en réalité «surjik», un mélange personnel d'ukrainien et de russe (Boyko, 2005).

La modernité joue contre l'ukrainien. Les livres écrits en russe couvrent $90 \%$ du marché, les publications en ukrainien n'ayant qu'un marché limité. La presse russe est présente partout, avec une vision plus internationale que la presse en langue ukrainienne, plus provinciale. Enfin, 98 \% des sites internet ukrainiens utilisent le russe (Teurtrie, 2010).

21 Si on se fonde sur la langue maternelle déclarée lors du recensement ukrainien, les 25 régions d'Ukraine ( 24 oblasts et une république autonome) se rattachent à six cas de figure (voir tableau 1).

Tableau 1- Langue maternelle déclarée par la population

\begin{tabular}{|l|l|l|}
\hline & langue maternelle & langue maternelle \\
\hline & ukrainienne & russe \\
\hline Crimée & & \\
\hline Donbass & $30 \%$ maximum & $69 \%$ et plus \\
\hline Sud et Est & $46-54 \%$ & $42-48 \%$ \\
\hline Bas Dniepr & $67-73 \%$ & $25-32 \%$ \\
\hline Haut Dniepr & $84-90 \%$ & $10-16 \%$ \\
\hline Centre & $92-93 \%$ & $6,5-7 \%$ \\
\hline Ouest & $95-98 \%$ & $1-5 \%$ \\
\hline
\end{tabular}

Donbass : Lougansk, Donetsk ; Centre-sud : Kharkov, Zaporojié, Odessa ; Bas Dniepr : Dniepropetrovsk, Mikolaïev, Kherson ; Haut Dniepr : Kirovabad, Poltava, Soumy, Tchernigov ; Centre : Kiev, Jitomir, Tcherkassy; Ouest: Vinnitsa, Khmielnitski, Tchernovtsy, Ternopol, Rovno, Volhynie, Lviv, IvanFrankivsk, Transcarpatie. La ville de Sébastopol, distinguée dans les statistiques ukrainiennes, a été regroupée avec la Crimée. De même, les données de la ville de Kiev sont fusionnées avec l'oblast du même nom.

Source : All Ukrainian population census 2001. 

comme langue maternelle (on les désignera sous le nom de "russophones »). La Crimée se rattacherait à ce groupe. Trois oblasts au centre et au sud présentent une situation de relatif équilibre entre russophones et ukrainophones. Trois oblasts du bas Dniepr ont de fortes minorités russophones (de un quart à un tiers). Trois oblast du haut Dniepr comptent entre 9,5 et $10,3 \%$ de russophones et une énorme majorité d'ukrainophones (89-90 \%). On y a rattaché l'oblast de Soumy, qui les jouxte, et qui ne diffère du schéma ( $16 \%$ de russophones pour $84 \%$ d'ukrainophones) qu'en raison de districts frontaliers. Trois oblasts centraux comptent 6-7 \% de russophones et 92-93\% d'ukrainophones. Dans les neuf oblasts de l'ouest, la part des ukrainophones dépasse $93 \%$, celle des russophones est partout marginale (3 à $5 \%$ ). Deux oblasts se distinguent. En Transcarpatie, 12,7 \% de la population se déclare de nationalité hongroise. Dans celui de Tchernivtsy, 17,5\% de la population se déclare de nationalité roumaine ou moldave, ce qui réduit la part des ukrainophones à respectivement 81 et $77 \%$. Ces oblasts correspondent aux régions historiques de Ruthénie subcarpatique et de Bucovine du nord.

actif, appuyé sur une forte diaspora aux États-Unis. Il réclame la reconnaissance d'une nationalité propre. Les ukrainophones, qui souffrent pourtant d'avoir été considérés comme des Polonais parlant mal le polonais puis comme des Russes parlant mal le russe, ne l'acceptent pas, expliquant qu'il n'y a pas de nationalité " ruthène ", mais des Ukrainiens qui ne parlent pas bien ukrainien (Maksimiuk, 2006).

La fragilité du pays se lisait sur la carte des élections présidentielles de 2010, opposant radicalement deux Ukraines.

\section{Carte 1 - Résultats au $2^{\mathrm{e}}$ tour de l'élection présidentielle de 2010}

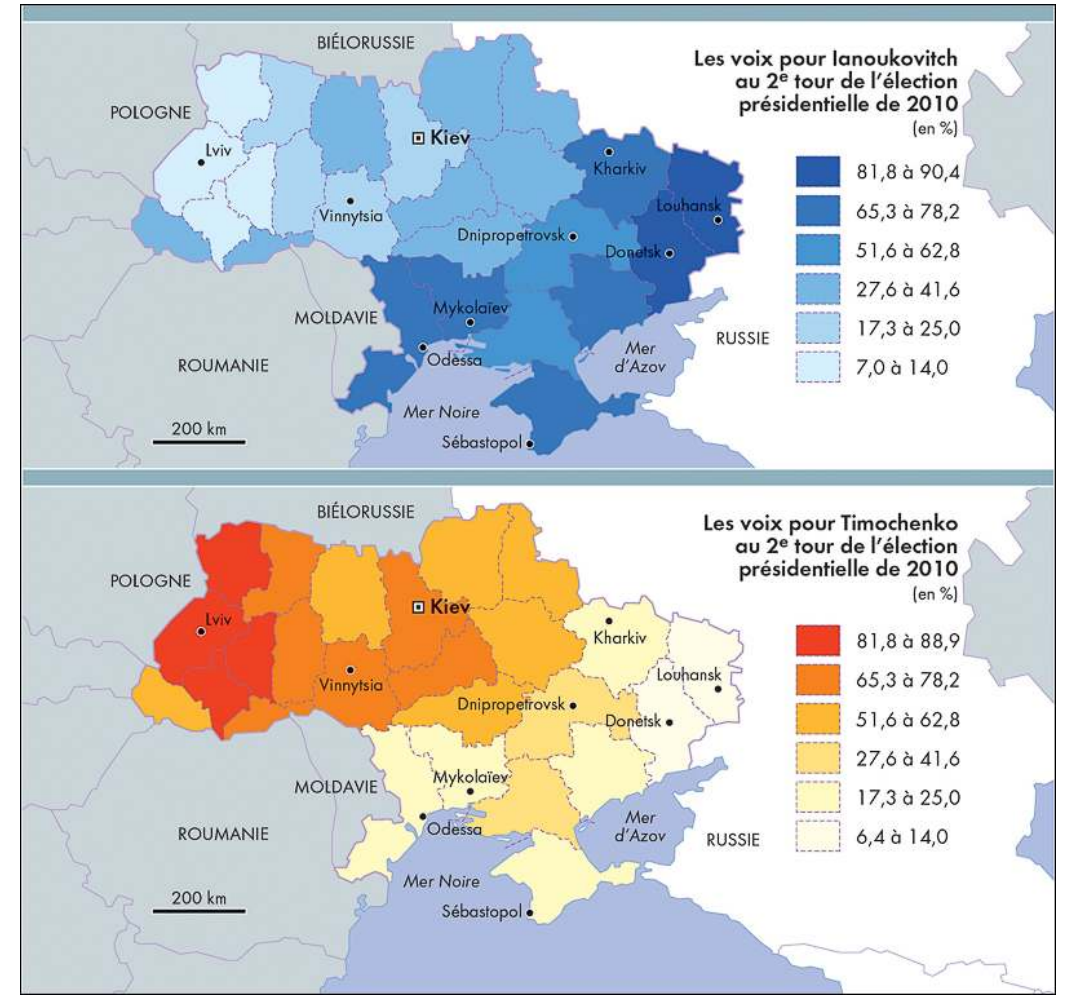

Source : P. Marchand, Atlas géopolitique de la Russie, Autrement, Paris, 2012. 
économiques ou stratégiques?

\section{Des enjeux spatialisés}

\section{Le poids économique relatif de ces ensembles est variable ${ }^{3}$}

Tableau 2 - Le poids économique des régions de l'Ukraine

\begin{tabular}{|l|l|l|l|l|}
\hline & surface & population & PIB & exportations \\
\hline Crimée & 4,5 & 5,2 & 3,7 & 1,6 \\
\hline Donbass & 8,8 & 14,5 & 15,7 & 25,2 \\
\hline Sud et Est & 15,2 & 15,2 & 13,8 & 11,5 \\
\hline Bas Dniepr & 14,1 & 12,2 & 13,5 & 19,5 \\
\hline Haut Dniepr & 18,1 & 10,2 & 8,7 & 8,1 \\
\hline Centre & 13,2 & 15,7 & 27,5 & 24,8 \\
\hline Ouest & 26,1 & 27,1 & 17 & 9,3 \\
\hline
\end{tabular}

Le Centre-sud (Kharkov, Zaporojié, Odessa), tant en part de PIB qu'en part d'exportations se situe en deçà de son poids démographique. C'est l'inverse pour le Bas Dniepr, qui est plus important en termes de PIB et surtout de poids dans les exportations, qu'en termes de population. Ceci est surtout dû à l'oblast de Dniepropetrovsk (15,5\% des exportations ukrainiennes à lui seul). Le Centre assure une part de PIB et d'exportations double de son poids démographique, surtout du fait du district urbain de Kiev, qui assure notamment $22,6 \%$ des exportations du pays. Il s'agit d'un effet "statistique » : une part de celles des autres régions est comptabilisée dans les directions de la capitale. Le Haut Dniepr et l'Ouest ont un poids économique très inférieur: avec $37 \%$ de la population, ils ne représentent que $25 \%$ du PIB et n'assurent que $17 \%$ des exportations. Le hiatus est surtout fort pour l'Ouest : $27 \%$ de la population mais seulement $9 \%$ des exportations.

Sur le plan des ressources minérales, l'Ukraine ne figure dans les dix premiers rangs mondiaux que pour trois métaux : le fer $\left(6^{\mathrm{e}}, 5 \%\right.$ de la production mondiale), le manganèse $\left(7^{\mathrm{e}}, 2,5 \%\right)$ et le titane $\left(8^{\mathrm{e}}, 5 \%\right)$. Ces productions sont principalement localisées dans le BasDniepr. Celle de charbon est concentrée à $82 \%$ dans le Donbass (Lougansk et Donetsk) mais l'Ukraine n'occupe que le $12^{\mathrm{e}}$ rang mondial pour ce produit ( $1 \%$ de la production mondiale). Les ressources en gaz de schistes (encore hypothétiques) seraient concentrées dans l'oblast de Kharkov et le Donbass.

En matière agricole, le nord de l'Ukraine (Ouest, Centre, Haut Dniepr) est essentiel avec $65 \%$ de la production de céréales, $95 \%$ de celle des betteraves à sucre, $37 \%$ du tournesol, $70 \%$ du troupeau de bovins. Le sud (Centre-sud et Donbass) ne joue un rôle que pour les céréales (35\% de la production) et le tournesol (60\% de la production). 


\section{Deux régions sont de réels enjeux}

\section{La Crimée, qui de facto n'est plus en Ukraine}

Son poids économique dans l'Ukraine était marginal. La réaction russe qui s'y est produite est uniquement liée à des motifs stratégiques.

Suite aux affrontements sanglants à Kiev, le 21 février 2014, trois ministres des affaires étrangères européens dépêchés en urgence avaient amené le président Ianoukovitch à un compromis avec l'opposition. Or l'accord est mis en pièce sous la pression des ultranationalistes de l'Ouest. En moins de 48 heures, le président élu en 2010 est destitué, le statut de la langue russe de 2010 est résilié, le gouvernement de coalition prévu est limité à la seule opposition.

$31 \mathrm{Vu}$ de Moscou, rien n'empêchait une dénonciation unilatérale des accords de 2010 sur Sébastopol ${ }^{4}$ aussi expéditive que celle du statut de la langue russe. Toute assurance des Occidentaux que les nouvelles autorités de Kiev n'iraient pas jusque-là était par avance discréditée par leur acceptation du viol immédiat d'un accord pourtant élaboré sous leurs auspices. La seule certitude de ne jamais voir la flotte de l'OTAN à Sébastopol était une prise de contrôle direct. Impensable avant le coup de force de Kiev, elle devenait la seule garantie absolue après.

32 Le scénario de l'annexion n'était pas celui envisagé au départ à Moscou, mais la réaction russe fut très rapide (Lévesque, 2014). De fait, le gouvernement russe a eu le choix entre deux mauvaises solutions : prendre le contrôle de la Crimée, avec l'assurance d'une crise internationale, ou risquer à tout moment de voir l'OTAN à Sébastopol.

Il suffisait pourtant de regarder la carte et de se remémorer l'histoire pour prévoir la réaction russe. Sans Sébastopol, le littoral russe de la mer Noire est étranglé ; avec, la Russie s'assure un "porte-avion » en plein cœur de la mer Noire et sécurise ses accès maritimes. 
Carte 2 - Carte géopolitique du bassin de la mer Noire

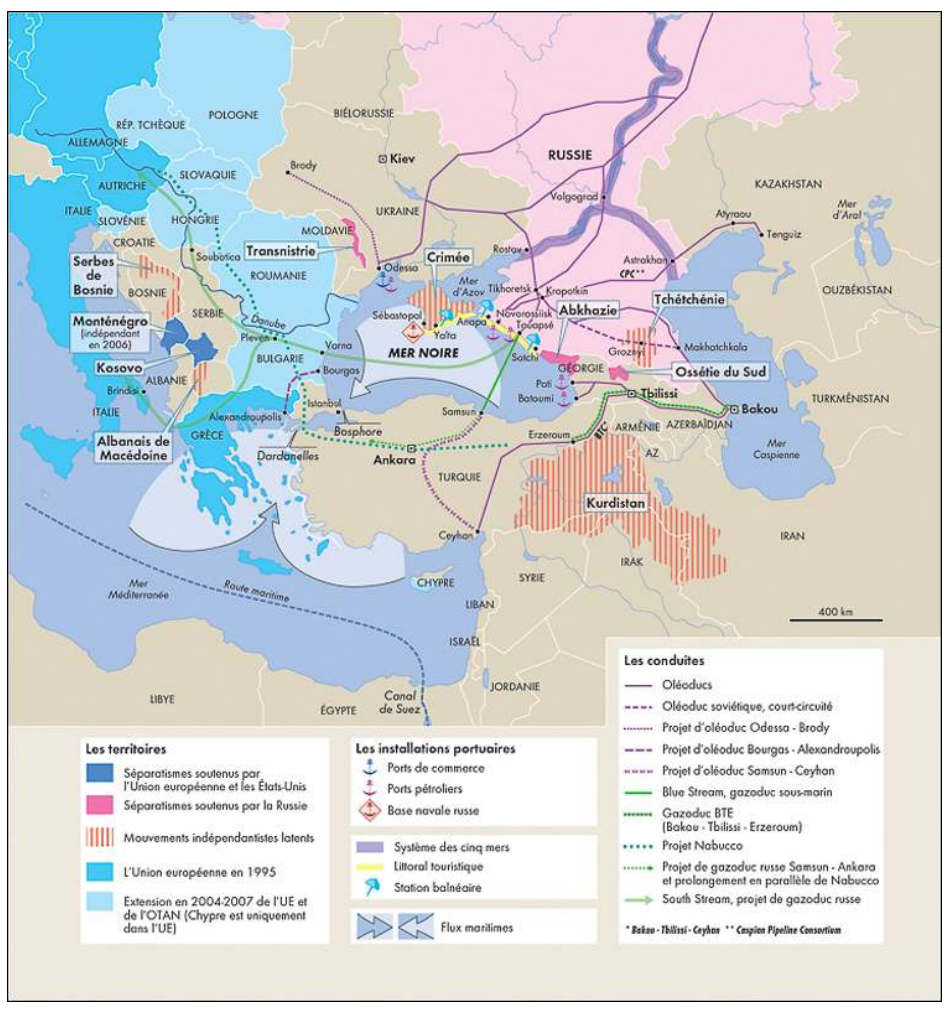

Source : P. Marchand, Atlas géopolitique de la Russie, Autrement, Paris, 2012.

\section{La question du Donbass}

Cette région assure le quart des exportations ukrainiennes en valeur. Cela peut sembler paradoxal puisque tous les reportages y montrent un appareil industriel vétuste et une population pauvre. L'industrie d'époque soviétique n'y survit que grâce aux subventions d'État. Ces subsides sont cependant attribués aux travailleurs en monnaie ukrainienne, en papier, alors que le produit de l'exportation est perçu en devises, mais par les dirigeants politiques et les oligarques. Le contrôle des oblasts de Donetsk et Lougansk est donc essentiel pour Kiev.

En revanche, le Donbass n'apporterait rien à la Russie dont elle ne dispose déjà en abondance (charbon, produits métallurgiques, excédent commercial) et il n'occupe pas une position clef sur la carte. Dans l'ordre stratégique, un autre élément est à considérer. Sans la Crimée, le poids des russophones en Ukraine est déjà réduit à 27,4 \% (contre 29,6 au recensement de 2001). Si les oblasts de Donetsk et de Lougansk s'excluaient, la part des russophones dans ce qui resterait de l'Ukraine serait ramenée à 19,7\%, ce qui représenterait un net affaiblissement de l'influence russe. Il est utile de ce point de vue pour Moscou que le Donbass reste dans une Ukraine fédéralisée.

Il y a donc des éléments objectifs pour comprendre la détermination de Kiev à garder le Donbass comme la réserve de Moscou face au séparatisme de cette région entre mars et juin. Dans le courant de l'été, deux éléments nouveaux sont intervenus : le succès des forces de Kiev face à des séparatistes mal armés a fait planer la menace de leur écrasement, et l'utilisation de l'artillerie et des bombardements aériens contre les villes a fait bondir le nombre de victimes, surtout civiles. Pour le Kremlin, qui a tant affirmé se 
poser en protecteur des populations russes, il en allait de sa crédibilité tant à l'extérieur qu'à l'intérieur même de la Russie.

Quelle que soit l'issue de la crise au Donbass, imprévisible à cause de l'importance que le facteur émotionnel y a pris, l'annexion de la Crimée pose à elle seule le problème de l'avenir des relations entre Kiev et Moscou.

\section{L'interdépendance économique avec la Russie}

L'analyse géopolitique de ce phénomène requiert un inventaire aussi précis que possible. Les données techniques et économiques qui serviront de base sont issues du dépouillement systématique de la presse économique française et russe (Les Echos, Kommersant, Ekspiert, Viedomosti), de publications spécialisées (Air\&Cosmos, Journal de la Marine Marchande, Défense \& Sécurité Internationale, Military Balance), des sites des associations professionnelles et des entreprises. Attendu le nombre de sources mobilisées (plus de 80 articles) il ne sera pas possible de détailler chacune d'entre elles ${ }^{5}$.

\section{Les liens technologiques avec la Russie}

Les RSS d'Ukraine et de Biélorussie avaient reçu une part importante de l'énorme complexe militaro-industriel qui travaillait à suréquiper l'armée soviétique. Les usines étaient intégrées dans des chaînes de production qui ne tenaient pas compte des limites administratives entre RSS. Les industries de pointe implantées en Ukraine (constructions navales militaires, matériel de guerre, aéronautique civile et militaire, industrie spatiale) étaient donc très développées, mais travaillaient en symbiose avec leurs homologues russes dans des programmes fédéraux. Avec l'implosion de l'URSS et l'apparition de frontières, les problèmes ont surgi immédiatement. La crise actuelle risque de déboucher sur une rupture totale.

\section{Les industries du matériel blindé et de la construction navale ont été frappées dès l'indépendance de 1991}

Le KB (bureau d'étude) Morozov et l'usine Malychev de Kharkov constituaient l'un des trois pôles de production de blindés de l'URSS. Dans la seule année 1991, l'usine avait produit 800 chars pour l'armée soviétique et les pays clients de Moscou.

41 Les gouvernements russes et ukrainiens ayant stoppé toute commande dans les années qutre-vingt-dix, comme ses homologues russes, l'usine s'est tournée vers l'exportation, mais la production s'est effondrée: entre 1992 et 1996, elle n'a produit en tout que 46 chars. Le KB Morozov a élaboré un nouveau modèle, le T-84, proposé sur les marchés internationaux à partir de 1999, et qui a obtenu des succès (Pakistan notamment), ainsi qu'une nouvelle version du T-72, le MP, élaborée en coopération avec la Slovaquie et la France. Il a également conçu une famille de blindés transport de troupes à roue, les BTR-4. Malgré cela, le complexe de Kharkov n'est plus que l'ombre de lui-même.

Le chantier naval de Mikolaïev, qui avait l'exclusivité de la production des porte-aéronefs soviétiques était d'une importance considérable (il employait près de 30000 personnes). En 1991, la construction du quatrième bâtiment de la classe Kiev, le "Bakou », y était en cours. Il a été achevé, mais par le chantier naval de Severo-Dvinsk, en Russie, et livré à la marine indienne en 2013. Si la France honore son contrat de livraisons de « Mistral » à la 
Russie, ce sont les chantiers navals de Saint Pétersbourg qui assureront la part russe du contrat.

De 1970 à 1990, les chantiers navals de Kiev et surtout de Kertch, en Crimée, ont assuré une partie de la production des frégates « Krivak » et des corvettes « Gricha » de la flotte soviétique. Là encore, les commandes ont disparu avec l'URSS. Depuis que la marine russe met à nouveau en service des navires de guerre (années 2000), toutes les coques ont été réservées aux chantiers russes. La marine ukrainienne étant insignifiante, les chantiers navals ukrainiens ont à peu près disparu, avec toutes leurs compétences technologiques. La seule unité de production encore à flot est le petit chantier naval de Feodosia, en Crimée, spécialisé dans les hovercrafts. Il assure notamment actuellement la production de navires d'assaut amphibie (le modèle le plus puissant existant au monde) pour la Chine et la Russie.

Coupées du marché de la Russie et de ses alliés, les industries du blindé et de la construction navale militaire, avec leur marché national réduit, ne peuvent espérer de salut que dans l'exportation. Or dans ce domaine, la concurrence est impitoyable et de très haut niveau, ce qui requiert des moyens financiers, que les entreprises ukrainiennes n'ont pas.

\section{L'industrie spatiale reste très liée à celle de la Russie}

Le KB Ioujnoïe et l'usine Ioujmach de Dniepropetrovsk jouaient un rôle clef dans la défense de l'URSS. Ils avaient été chargés de concevoir et produire le premier IRBM ${ }^{6}$ soviétique standard, le SS-4 (celui de la crise de Cuba en 1962). Servant aussi de premier étage de propulsion au lanceur spatial "Cosmos", assemblé à Omsk, en Russie, il fut produit à 2300 exemplaires.

Ultérieurement, ce complexe s'est vu attribuer la conception et la construction du plus puissant ICBM soviétique, le SS-18. En 1986, 308 des 1418 ICBM $^{7}$ soviétiques étaient des SS-18. En 2013, le parc de 356 ICBM russe compte encore 54 SS-18. En 1991, Ioujmach était retenu pour construire l'ICBM qui forme l'ossature de la force nucléaire stratégique russe actuelle, le Topol M, tâche qui lui a échappé avec l'éclatement de l'URSS. Il assurait toujours la maintenance des SS-18 russes, qui lui a été retirée au printemps 2014.

Ioujmach avait par ailleurs le monopole du recyclage de 150 SS-18 en lanceur de satellite civil « Dniepr », tiré à partir de la base de Iasnyi en Russie. Depuis 2011, les autorisations russes de lancement de "Dniepr" s'étaient raréfiées pour favoriser les lanceurs nationaux. Le programme russe de lancements ayant subi accidents et retards, pour éviter une fuite de la clientèle, Ioujmach avait cependant reçu des autorisations de tir pour le second semestre 2013 : un tir a eu lieu en août, un autre en novembre. Deux étaient prévus en 2014, dont l'avenir est compromis.

Ioujnoïe-Ioujmach a conçu, construit et commercialise encore l'un des trois lanceurs spatial lourd soviétique, le "Zenit» (les deux autres étant les russes "Proton» et « Soïouz »). Le « Zenit » associe deux étages de propulsion ukrainiens et un russe et lance des satellites pour tous clients internationaux à partir de Baïkonour (Kazakhstan) ou Plessetsk (Russie).

$50 \mathrm{Au}$ début des années quatre-vingt-dix, pour pouvoir lancer à partir d'une position équatoriale, donc satelliser une charge plus lourde, Ioujnoï-Ioujmach (15\%), s'est associé avec le russe Energia (25\%), le constructeur naval norvégien Kvaerner $(20 \%)$ et Boeing 
(40\%), dans le programme Sea Launch. Les éléments de fusées "Zenit» construits en Ukraine et en Russie sont assemblés en Californie. Le lanceur est installé sur la plateforme flottante qui va se positionner dans le Pacifique à la latitude requise. Après l'explosion d'une fusée sur la plate-forme, Sea Launch a été mis en faillite puis racheté par Energia (95\% des parts, Kvaerner et Boeing s'en partageant $5 \%$ ). En mai 2014, sa situation est délicate, car le carnet de commande se limite à un tir pour 2016 (pour des satellites russes).

Avant le SS-18, le groupe avait produit depuis 1967 l'ICBM SS-9. À partir de cet engin, il a extrapolé un lanceur civil plus léger, le « Tsyklon » (versions I, II et III), opérant à partir de Plessetsk (Russie). Quelques « Tsyklon III » ont encore été tirés en 2013. Les systèmes de guidage sont produits à Kharkov et Kiev. Le groupe travaille à une nouvelle version, le "Tsyklon IV », dans le cadre d'un programme ukraino-brésilien. Il serait tiré à partir du cosmodrome d'Alcantara (Brésil). Ioujnoïe participe également au programme de lanceur léger italien «Véga », dont il fabrique l'étage de propulsion supérieur.

Ioujnoïe-Ioujmach n'employait plus que 13000 personnes en 2010 (contre 62000 en 1991). Son importante production de satellites d'époque soviétique (plus de 70 ont été produits) est également en déconfiture. Autre fleuron soviétique, Elektropribor, à Kharkov fabriquait les systèmes de contrôle de nombreux ICBM et lanceurs soviétiques, ainsi que du « Zarya » (module russe de l'ISS). Il a construit plus de 150 satellites « Cosmos » pour le compte de Moscou. Privé de ces contrats hautement stratégiques, le groupe a été racheté en 1994 par l'américain Westinghouse. Il développe le système de guidage du « Tsyklon IV ».

Ioujnoïe-Ioujmach est le pilier de l'industrie spatiale ukrainienne. Les "Zenit », lancés de Baïkonour (huit par an) ou par mer et les «Dniepr», lancés de Russie, sont les pourvoyeurs en devises. La rupture avec la Russie va tarir ces moyens de financement pour le projet «Tsyklon IV » et ruine son projet d'intégrer le programme «Baïterek » (version commerciale kazakho-russe du lanceur «Angara» russe, successeur du «Proton »). On voit mal comment le groupe pourra fonctionner sans l'accès aux cosmodromes russes et à Baïkonour et le basculement dans le camp occidental paraît difficile, Union Européenne et États-Unis ayant assez de difficultés à financer leur secteur spatial pour ne pas subventionner le maintien sur le marché d'un concurrent.

\section{L'industrie aéronautique est en grande difficulté}

En 2010, l'industrie aéronautique emploie encore 68000 salariés dans 39 entreprises. Mais l'important constructeur d'époque soviétique, Antonov, est en bout de course : en 3 ans, de 2010 à 2012, il n'a plus produit que six avions. Sa production repose sur trois modèles, dont deux sont d'époque soviétique, surtout vendus dans la CEI :

- L'An 140 est un bimoteur léger. Au $1^{\mathrm{er}}$ novembre 2013, 31 seulement ont été vendus. Hors d'Ukraine, il n'a été exporté qu'en Russie et en Iran.

- L'An 72-74, biréacteur léger, a été construit à 80 exemplaires, dont une cinquantaine volent encore fin 2013 (en Ukraine, Russie, Iran, Egypte, Arménie).

- L'avion régional An 148 a été conçu à la fin des années 90. Il avait obtenu plusieurs dizaines de commandes, mais dès 2004, l'usine de Kharkov s'avérait incapable de livrer ses éléments (caisson central, nacelle moteur...). La production a été recentrée sur deux chaînes de production du groupe, celles de Kiev et de Voronej (Russie). C'est ensuite l'usine de Kiev dans laquelle se sont englouties les subventions sans que les appareils ne sortent des 
chaînes. Seule l'unité de Voronej fonctionne tant bien que mal. Devant ces défaillances, la plupart des commandes ont été annulées. En juillet 2013, moins de 20 appareils étaient en service, dont 11 en Russie, 4 à Cuba, 2 en Ukraine et un en Corée du Nord.

Deux projets d'Antonov intéressaient la Russie. Une autre usine du groupe se trouve en Russie, à Oulianovsk. Elle a assuré (pour 2/3) avec l'usine de Kiev (pour 1/3), la production $\mathrm{du}$ très gros porteur An-124. Cet avion-cargo est la pièce essentielle de toutes les opérations de l'ONU et de l'OTAN depuis deux décennies. Gérés par une compagnie russe et une ukrainienne, ils ont entre autre, effectué plus de 600 missions entre 2002 et 2007 pour les forces américaines en Irak. Ce sont eux qui rapatrient actuellement le matériel français d'Afghanistan et ont permis le déploiement au Tchad ou en Centrafrique. Le bureau d'étude des gros porteurs Antonov est à Oulianovsk où, depuis 2010, la Russie voulait relancer la production. Le projet achoppait sur le fait que les usines ukrainiennes $\mathrm{du}$ groupe ne sont plus en mesure de livrer les parties d'avion dont elles sont responsables. Il n'a plus de chances de voir le jour.

L'An 70 est un avion de transport militaire à la capacité d'emport un peu supérieure à celles de l'A $400 \mathrm{M}$. Il devait être commandé à 150 exemplaires par l'Armée de l'air russe, mais ce contrat a été annulé en 2006 après la « révolution orange ». En 2010, il a été signé à nouveau pour 60 exemplaires. L'avion devait être assemblé à Kazan (en Russie) à partir d'éléments en partie produits en Ukraine (voilure, nacelles moteur, pylônes). La réalisation de ce projet paraît exclue.

L'autre pilier de l'industrie aéronautique ukrainienne est Motor Sitch, de Zaporojié (22 000 employés en 2010), qui produit des moteurs et des réacteurs. Il est très impliqué dans le secteur des hélicoptères, dont la Russie est un des principaux producteurs mondiaux: presque tous les modèles produits en Russie peuvent être équipés d'un moteur Motor Sitch. Ce serait de fait le cas de $70 \%$ du parc. Le motoriste ukrainien équipe également une version du nouvel avion d'entrainement standard russe, le Iak 130, et les hydravions russes Beriev 200, proposés sur le marché international comme appareil de lutte contre les incendies.

Après la « révolution orange ", la révision annuelle de 600 moteurs d'hélicoptères avait déjà été retirée à Motor Sitch mais un accord de 2011 lui avait restitué le travail pour 250 moteurs (contrat sur 5 ans pour 1,5 milliards de \$). Depuis mars 2014, les autorités russes imposent que les hélicoptères russes reçoivent dorénavant des moteurs nationaux. Les motoristes russes vont donc augmenter leur capacité de production. D'autres débouchés vont se fermer: Motor Sitch était pressenti pour motoriser le drone MALE russe et pour participer à la construction du réacteur du moyen-courrier russe MS 21 . Il devait motoriser une partie des An 70 achetés par la Russie. Motor Sitch ne conserve assurément que la motorisation des avions produits en Ukraine, soit quelques unités par an, ainsi que des An 148 assemblés à Voronej. L'avenir du groupe paraît délicat car on imagine mal que les hélicoptéristes occidentaux rompent avec leurs fournisseurs pour lui offrir des débouchés.

\section{D'autres industries ukrainiennes de haute technologie travaillent pour le marché russe}

59 Une partie des équipements de ventilation, des armatures et des tubulures des nouvelles centrales nucléaires russes VVER est fabriquée en Ukraine dans des usines situées à Kiev, 
Ivan-Franko et Kramatorsk (Rosatom est propriétaire à $51 \%$ de cette dernière). La question du transfert des commandes vers des producteurs russes est posée.

Les turbines à gaz de Zorya Mashproekt de Mykolaïev équipent les frégates russes "Admiral Gorshkov» d'un modèle fabriqué sous licence en Russie, à Iaroslavl. Zorya devait livrer les turbines pour équiper les nouvelles frégates russes «Admiral Grigorievitch », accord menacé. Zorya produit et exporte aussi de par le monde des turbines pour les gazoducs et les champs d'exploitation gaziers. À ce titre, elle devait participer à la mise en valeur en cours des gisements de Iamal en Russie.

\section{Le poids incontournable de la situation géographique}

61 Par sa situation géographique, l'Ukraine est un couloir naturel entre l'Eurasie et l'Europe de l'ouest, atout économique enviable. Les empires russes et soviétiques y avaient concentré leur appareil portuaire de la mer Noire. Le régime soviétique y avait tracé le gazoduc acheminant le gaz sibérien vers l'Ouest. Une rupture entre les deux pays entraînerait un tarissement des flux à travers l'Ukraine.

\section{L'appareil portuaire}

62 En 1990, dernière année soviétique, les ports ukrainiens avaient manipulé 62 millions de tonnes, plus que leurs homologues russes de la mer Noire (58) (Bernard, 1991). En 2012, leur trafic atteint 96 millions de tonnes, celui des ports russes, 172 millions de tonnes : le trafic de la façade russe est deux fois supérieur à celui de son homologue ukrainienne. La Stratégie 2030 de la Russie prévoit de le porter à 290 millions de tonnes, grâce à la création du port de Taman ( 2 millions de tonnes en 2012 pour sa première année de fonctionnement, 100 millions prévues pour 2020) (Marchand, 2014).

63 Toutefois, la Crimée dispose de quatre ports en eau profonde. Le développement de deux d'entre eux suffirait à assurer celui qui était prévu à Taman, et sur des sites offrant de meilleures qualités nautiques. Ceci cependant à condition d'assurer le franchissement du détroit de Kertch. Le choix a été rapidement opéré : il s'agira d'un pont à quatre voies de circulation routière à péage et deux voies ferroviaires. La construction doit être réalisée par des entreprises russes et chinoises pour 2018. En raison du tracé choisi, les emprises terrestres nécessaires à l'accès du pont vont occuper celles prévues pour les installations du port de Taman, dont l'extension est abandonnée. Des investisseurs privés envisagent néanmoins d'en réaliser une partie à leurs frais. Il n'y a pas encore de précision sur la hauteur du pont, mais s'il laisse un faible tirant d'air, il entravera voire bloquera l'activité de Marioupol, $4^{\text {e }}$ port ukrainien (15 millions de tonnes en 2013).

\section{Le transit gazier à travers l'Ukraine}

Selon Gazprom, en 2013, 86 milliards de $\mathrm{m}^{3}$ de gaz destinés à l'UE ont transité à travers l'Ukraine, 30 à travers la Biélorussie, 24 par North Stream (qui a pourtant une capacité de 55).

Moscou exige depuis $1^{\mathrm{er}}$ avril que Kiev paye le gaz qu'elle achète ( 28 milliards de $\mathrm{m}^{3}$ en 2013) au prix de $485 \$$ les $1000 \mathrm{~m}^{3}$, soit le montant le plus élevé de tout le continent européen. Kiev n'accepte que $268 \$^{8}$, et tant qu'elle ne l'a pas obtenu, refuse de payer le gaz russe qu'elle consomme. La négociation est au point mort, mais pour le FMI et la 
Commission européenne $(\mathrm{CE})$, Kiev ne pourra obtenir un prix inférieur à celui du marché spot en Europe (300-380 \$ pour $1000 \mathrm{~m}^{3}$ au cours de l'hiver 2013-14).

Moscou menaçait depuis avril de ne plus livrer à l'Ukraine que le gaz préalablement payé. Un premier ultimatum avait été fixé au $1^{\mathrm{er}}$ juin, date étonnamment tardive, puis repoussé au 10 , puis encore au 16, date à laquelle il a finalement été appliqué. Le montant des impayés à cette date s'élevait à 4,2 milliards de \$ pour la seule période novembre 2013juin 2014.

Cette modération russe est étonnante. L'Ukraine doit en effet, pour couvrir les superpointes de consommation de l'Europe cet hiver, compléter ses réservoirs souterrains de gaz avant octobre. Début mai, 17 milliards de $\mathrm{m}^{3}$ étaient nécessaires pour cela. En conditionnant la livraison du gaz à son paiement préalable, le Kremlin aurait empêché Kiev de compléter les réservoirs. Pourtant, il l'a laissé pomper sans payer jusqu'au 16 juin. Quelques éléments éclairent cette étrange séquence :

- Le Commissaire européen à l'énergie avait déclaré à la presse russe que «la guerre civile à l'Est et la non-reconnaissance du gouvernement de Kiev n'était pas favorable au projet South Stream ». En clair, derrière cette subtile formulation diplomatique, la CE, qui depuis plusieurs années s'oppose par tous les moyens à ce projet de gazoduc, laissait entrevoir à Moscou un assouplissement de son opposition en échange d'une contribution russe à une normalisation en Ukraine.

- Le 7 juin, une délégation de trois sénateurs envoyée par Barack Obama (dont John Mac Cain) a rencontré le premier ministre bulgare à Sofia. Le lendemain, ce dernier annonçait que la Bulgarie, point d'arrivée du gazoduc South Stream, renonçait à ce projet. On ignore les arguments qui ont convaincu le premier ministre bulgare de renoncer à un gazoduc qui apporterait un à deux milliards de dollars par an de droits de passage à son pays, subventionné par les fonds structurels financés par le contribuable européen à hauteur de quelques milliards de dollars par an. En tout cas, la CE n'avait plus rien à laisser espérer à Moscou. Confirmation obtenue, Gazprom a mis son ultimatum à exécution le 16 juin.

- En vertu de ses contrats, Gazprom est tenu de livrer le gaz à ses clients ouest-européens et ne peut se retrancher derrière un problème de transit. En 2009, Gazprom a ainsi dû payer 3 milliards de dollars de dédommagement pour le gaz prélevé par l'Ukraine et qui n'était pas parvenu aux clients occidentaux. Gazprom a donc aidé ces derniers à remplir au maximum leurs stockages souterrains en prévision de difficultés cet hiver: fin août les réservoirs souterrains d'Europe de l'Ouest étaient remplis à $87 \%$ de leur capacité, niveau record pour cette date. Le retard dans l'application du pré-paiement a participé à cette stratégie.

68 A court-terme, la question du transit du gaz destiné à l'UE reste néanmoins posée. En 2009, Gazprom avait réduit ses envois de la quantité destinée à l'Ukraine, mais celle-ci avait prélevé pour ses besoins sur le volume en transit. Or, Gazprom, par son contrat de type ship-or-pay avec la Slovaquie, est obligé de faire transiter la quantité de gaz ou de payer comme si cela avait été le cas. Ceci explique qu'en 2013 encore, Gazprom ne puisse pas utiliser pleinement la capacité de North Stream : le groupe est contraint par contrat d'assurer un volume de transit par la Slovaquie, donc l'Ukraine.

Pour l'hiver 2014-15, la CE travaille à organiser une inversion du flux de gaz vers l'Ukraine à partir de l'Allemagne, à travers la Hongrie et la Pologne. De 2 milliards de $\mathrm{m}^{3}$ par an depuis 2012, moyennant quelques investissements, elle pourrait porter sur 7 milliards de $\mathrm{m}^{3}$. La CE fait pression sur la Slovaquie pour qu'elle fasse fonctionner en sens inverse un des quatre tubes acheminant le gaz russe vers l'Ouest. Si elle accepte, le flux atteindrait 15 milliards de $\mathrm{m}^{3}$ (encore loin des besoins de l'Ukraine, 28 milliards en 
2013). Mais en vertu du contrat "ship-or-pay», la Slovaquie est, elle aussi, tenue de faire transiter la quantité dite de gaz russe, ou de payer des pénalités.

70 A moyen terme, le gouvernement de Kiev étudierait la possibilité de privatiser le réseau de gazoducs ukrainiens en faveur d'entreprises occidentales. Il n'en tirerait un bon prix que si ledit réseau reste un vecteur de transit vers l'Ouest. Or, South Stream réduirait cette fonction aux volumes prévus par les contrats "ship-or-pay", et seulement jusqu'à leur expiration. Bruxelles et Washington ont donc intérêt, pour favoriser Kiev, à ce que ce gazoduc, contournant l'Ukraine, ne soit pas réalisé.

71 Cependant, Gazprom a engagé la pose de ce tube au fond de la mer Noire depuis décembre 2012. Ankara lui offre une solution. La Turquie ne veut plus voir la moitié de ses achats de gaz russe menacés par le transit ukrainien (13 milliards de $\mathrm{m}^{3}$ sur 26 en 2013) et a demandé à Gazprom en avril 2014 de doubler Blue-Stream, tube sous-marin direct entre Russie et Turquie, d'ici deux à trois ans. Elle propose aussi à Gazprom d'étudier un autre tracé de South Stream, par son territoire et au-delà par des pays moins sensibles aux pressions américaines, comme l'Italie, qui a récemment refusé de retirer ENI du projet.

72 La question est donc complexe et la presse économique russe abonde en détails sur les arguments juridiques que fourbissent actuellement la CE, Kiev et Gazprom en vue de la longue partie d'échecs gaziers qui va commencer cet hiver. Mais l'écheveau des relations économiques Ukraine-Russie est compliqué par d'autres éléments encore.

\section{Le futur de l'Ukraine}

\section{L'association à l'UE}

73 Comme l'explique depuis longtemps Moscou, l'accord de libre-échange entre UE et Ukraine lui pose un problème. Les marchandises circulant librement à travers la frontière entre la Russie et l'Ukraine, un accord de libre-échange UE-Ukraine, permettrait aux produits de l'UE, par Ukraine interposée, d'entrer en Russie en contournant toutes les réglementations russes. Pourtant, la CE a toujours refusé d'impliquer la Russie dans les pourparlers, même après le coup de force de Kiev ou la crise de Crimée. L'accord du 27 juin va imposer à la Russie de revoir le statut de sa frontière avec l'Ukraine.

Quelles perspectives offre l'intégration de l'Ukraine à l'espace Ouest-Européen ?

Sur un plan général, on voit bien l'intérêt d'un soutien financier. À condition qu'elle ne soit pas détournée par les oligarques, cette assistance peut soutenir un développement économique. À supposer que le budget européen le permette, ce qui est très loin d'être assuré, dans quelle direction pourrait-elle s'orienter?

76 L'afflux spontané d'IDE en Ukraine est peu vraisemblable. Comme l'expliquent les investisseurs industriels déjà présents, l'intérêt du pays était sa situation de plate-forme d'exportation vers la Russie, avantage qui disparaît en cas de libre-échange UE-Ukraine. Le maintien d'une situation de conflit avec la Russie n'est au surplus pas de nature à les rassurer.

77 À terme, l'Ukraine pourrait devenir une base de production à bas coût. L'obstacle est que, sans perspective d'exportation vers le continent eurasiatique, le seul débouché est constitué par les marchés d'Europe occidentale, situés à l'autre extrémité du continent. 
78 Le ministre français du commerce extérieur déclarait en janvier 2014 que l'Ukraine est « un important débouché potentiel pour nos produits industriels ». Elle peut également remplir la fonction de pourvoyeur de matières premières. Cette répartition coloniale du travail offre toutefois peu de perspectives à l'économie ukrainienne.

79 Si des pertes de marché russes s'ajoutent à ces éléments, les perspectives de l'économie ukrainienne sont assez sombres. On comprend que la CE ait à plusieurs reprises invité Moscou à participer à son soutien.

80 En conflit avec Kiev, la Russie a-t-elle intérêt à prendre sa part du «fardeau ukrainien » comme l'y invite la CE ? Ou a-t-elle intérêt à suivre l'exemple des Etats-Unis qui, en 1962, ont placé leur voisin Cuba, qui s'était doté d'un gouvernement qui n'était pas de leur goût, sous un embargo qui dure depuis 50 ans?

\section{Quelle ampleur pour la réaction russe ?}

81 Le commerce extérieur ukrainien est plus lié à la CEI qu'à l'UE et serait très sensible à une rupture commerciale avec la Russie comme le montre le tableau 3.

Tableau 3 - Les orientations géographiques du commerce extérieur ukrainien

\begin{tabular}{|l|l|l|}
\hline 2012 & Exportations & Importations \\
\hline CEI & $36,77 \%$ & $40,69 \%$ \\
\hline UE & $24,82 \%$ & $30,89 \%$ \\
\hline Asie & $25,69 \%$ & $20,24 \%$ \\
\hline
\end{tabular}

Source : Annuaire statistique ukrainien

82 Au niveau russe, la dépendance est beaucoup plus réduite puisque l'Ukraine n'absorbe que $6 \%$ des exportations russes, dont beaucoup de gaz et de pétrole.

\section{Deux raisons contraignent assurément la Russie à restreindre ses importations d'Ukraine}

83 Le simple fait qu'un rapprochement entre Kiev et l'OTAN ne puisse être totalement exclu impose à la Russie, pour des raisons de sécurité, d'éliminer toute dépendance de son appareil militaire vis-à-vis d'un fournisseur ukrainien. Au-delà des cas exposés (entretien de missiles stratégiques, motorisation d'hélicoptères de combat ...), l'industrie ukrainienne livre un nombre important de composants pour l'industrie militaire russe. Rompre ces liens est un impératif stratégique pour la Russie. En juin 2014, le ministère russe de la Défense a annoncé étudier en détail avec son homologue biélorusse l'éventail de tous les transferts de production possibles depuis l'Ukraine vers l'industrie biélorusse.

La question de la survie de l'industrie aérospatiale ukrainienne, qui représente l'essentiel de l'industrie de haute technologie du pays, avec 100 à 150000 emplois très qualifiés, est donc posée. Dans ces domaines stratégiques, les pays développés, dans un contexte de réduction générale des commandes militaires, n'ont pas de place à offrir aux entreprises ukrainiennes, sauf à réduire les débouchés de leur propre industrie. L'aérospatiale 
ukrainienne peut se reconvertir partiellement en bureau d'études low-cost et fournisseur de pièces pour l'industrie occidentale (mais au détriment de ses sous-traitants actuels), très loin du rôle éminent qu'elle jouait dans le partenariat avec la Russie.

Par ailleurs, en raison de sanctions occidentales, Moscou doit soutenir par tous les moyens l'activité en Russie. La part du commerce international russe qui est confiée aux ports des pays voisins (ukrainiens et baltes) devrait être transférée vers les ports russes. Une politique de substitution des importations devrait également être mise en place, favorisant la production locale. Elle pourrait viser particulièrement les fournisseurs ukrainiens mais comme on l'a vu pour le nucléaire, le fait que certaines usines produisant en Ukraine pour le marché russe sont la propriété de groupes russes complexifie les choses.

Une rupture avec la Russie compromettra inévitablement deux piliers de l'activité ukrainienne: son industrie de haute technologie et sa fonction portuaire pour l'hinterland eurasien. A cela il faut ajouter la perte inéluctable des revenus du transit du gaz (3,5 milliards de dollars en 2013) : le contournement du pays est au dire du secrétaire du Fonds pour la sécurité énergétique de Russie « une question de principe » à réaliser d'ici quelques années.

\section{Au-delà de ces impératifs, Moscou peut moduler le degré de fermeture de la frontière}

Un blocage sélectif, ciblant les oblasts ukrainiens de l'Ouest est-il possible?

Les six oblasts dont les exportations sont le plus orientées vers la CEI se trouvent aussi bien à l'est (Kharkov, 71,05\%; Lougansk, 70,35\%), qu'au centre (Souma, 65,57\%; Tchernigov, 61,65 \%) ou qu'à l'ouest (Khmelnitsa, 53,66 \% ; Vinnitsa, 52,93 \%).

Ceux dont les exportations sont le moins dirigées vers la CEI sont ceux de Transcarpathie $(9,54 \%)$ et Lviv $(21,82 \%)$, à l'Ouest, mais aussi d'Odessa $(23,38 \%)$ et Donetsk $(25,9 \%)$, en zone russophone (Donetsk exporte davantage vers l'UE (26,7 \%) et l'Asie (36,12 \%)).

Moscou pourrait donc difficilement élaborer une stratégie de rétorsion commerciale « régionalisée » : à l'Est comme à l'Ouest de l'Ukraine, des oblasts sont peu dépendants des exportations vers la CEI, d'autres le sont beaucoup.

1 Même limitée aux nécessités stratégiques, la perte de marchés russes va provoquer des difficultés sociales en Ukraine. Les zones affectées, outre l'oblast de Lougansk déjà en rébellion, seront les centres d'industrie aérospatiale (Kiev, Kharkov, Dniepropetrovsk, Zaporojié), les façades portuaires (Odessa, Mikolaïev), les oblasts frontaliers très dépendants du marché russe pour leurs exportations (Kharkov, Souma, Tchernigov), toutes régions qui ne participent pas actuellement au mouvement dit " pro-russe ».

2 On ne peut pas encore préjuger de l'intensité des mesures de rétorsion russes. Elle va dépendre de l'évolution des relations entre les deux capitales et aussi des négociations avec la CE qui a enfin convenu en septembre que l'accord d'association avec l'Ukraine concernait aussi la Russie et a reporté son application à décembre 2015.

La question de fond est de savoir si la Russie peut trouver intérêt à ménager un pays qui veut lui tourner le dos. Or on ne voit pas ce qu'elle aurait à perdre à ce que ce voisin soit en crise. D'autant que les relations entre les deux pays seront mauvaises de toute façon, ne serait-ce qu'en raison de la Crimée. 

simples nécessités stratégiques russes, va aggraver la situation économique de cette dernière. Ceci conduit à une évidence : le coût du sauvetage de la Grèce n'a été qu'une «bagatelle», selon l'expression du commissaire européen Günther Oettinger, comparé aux besoins de l'Ukraine, qui nécessitera selon lui les efforts « de la CE, des États-Unis, du Canada, du FMI ». Il est d'ailleurs probable que tenir à bout de bras l'économie d'un pays de 45 millions d'habitants coupé de son hinterland naturel sera hors de portée de cette association, elle-même en situation économique précaire.

\section{Une internationalisation du conflit ? Le rationnel et l'irrationnel}

En raisonnant de façon rationnelle, le conflit n'est pas sans intérêt pour les Occidentaux :

- l'OTAN achève sa mission en Afghanistan. Il serait logique que l'institution, pour justifier la pérennité de ses services et de son budget, ne voie pas d'un mauvais œil la tension entre Kiev et Moscou, voire soit tentée d'œuvrer à l'entretenir ;

- dans l'UE, certains se souviennent que pendant la guerre froide, confrontée à la menace soviétique, l'opinion européenne ne contestait pas la construction européenne. La perception par les peuples européens d'un ennemi à l'Est permettrait de générer un " Autre ", face auquel pourrait renaître un sentiment européen en voie d'extinction;

- Washington ne peut que se réjouir de voir les pays européens inquiets. Compte tenu de leur dépendance en matière de sécurité, ils ne pourront qu'être plus accommodants avec les volontés de la Maison Blanche.

Le Kremlin est un acteur rationnel. Préoccupé du développement économique de la Russie, une fois la Crimée sécurisée, il n'a pas d'intérêt à un conflit l'exposant à des sanctions réelles. Cependant, à partir d'un certain seuil, il lui serait impossible de ne pas réagir militairement. Ainsi, si Kiev ne peut reprendre le contrôle de l'Est sans un massacre de séparatistes, quel sera le seuil de tolérance de l'opinion russe ? La CE aborde l'association de la Moldavie sur des bases radicales : la seule issue laissée aux autorités de Transnistrie est la capitulation sans conditions. Le Kremlin peut-il abandonner plusieurs centaines de milliers de russophones sans perdre la face vis-à-vis de son opinion publique ?

Il faut aussi tenir compte des données stratégiques. Ce qui se passe en Ukraine, c'est-àdire à sa porte, affecte la sécurité de la Russie. Elle ne peut rester aussi indifférente que, par exemple, la France vis-à-vis d'évènements se produisant au Mali ou en Centrafrique, à des milliers de kilomètres. Une réaction militaire russe de très grande ampleur est possible. D'autant que les passions sont tellement exacerbées dans le Donbass que l'évolution de la situation y est imprévisible.

Les médias occidentaux se sont convaincus que les sanctions expliquent que Moscou n'ait pas annexé le Donbass comme la Crimée. On a vu qu'il y d'autres raisons. Penser que la menace de sanctions a suffi et suffira à imposer les vues occidentales à Moscou est dangereux. Bien sûr, de véritables sanctions, frappant les hydrocarbures, les apports de technologie occidentale, la finance, affecteraient gravement l'économie russe, mais pas seulement elle. En effet la technologie asiatique (Chine, Japon, Corée, Inde ...) vaut aujourd'hui celle de l'Europe et peut la remplacer. Pour l'économie de l'UE, La perte consécutive de contrats en Russie aura des conséquences directes et une crise économique en Russie réduira le pouvoir d'achat dans ce pays, donc, indirectement les 
exportations de l'UE. Enfin, dès que les sanctions deviendront dommageables, des contremesures russes pourraient au surplus fermer des marchés.

Pour l'Europe de l'Ouest entrer dans le jeu de sanctions réelles contre la Russie présente un risque économique réel. On comprend que les gouvernements européens hésitent à céder aux pressions des États-Unis, dont l'économie ne subira quant à elle aucune conséquence. Surtout qu'une crise économique majeure provoquée par des sanctions animées par les États-Unis ouvrirait de larges perspectives à la diplomatie russe en Europe. Sur un plan général, il est de toute façon inapproprié de chercher dans une spirale de sanctions économiques incontrôlable la solution à un problème qui est d'ordre politique.

\section{Conclusion}

La CE s'est obstinée à obliger l'Ukraine à choisir entre Bruxelles et Moscou, alors que, compte tenu de la fragilité de ce pays, la voie de la sagesse était de ménager une solution permettant son association, et à l'UE, et à l'Union eurasiatique. A la simple vue du résultat des élections présidentielles ukrainiennes de 2010, nul ne pouvait ignorer la fragilité du pays.

101 Si cette voie avait été choisie par la CE, il n'y aurait pas eu de coup de force à Kiev, les élections présidentielles de décembre 2014 se seraient déroulées normalement, la Crimée serait encore en Ukraine, qui ne serait pas en guerre civile. L'affrontement entre un bloc atlantique reconstitué et la Russie n'aurait pas été porté à ce dangereux paroxysme, dommageable pour toutes les parties (Russie, Ukraine, UE), à l'exception des États-Unis.

\section{BIBLIOGRAPHIE}

Allain A., 2014. OTAN-Russie : l'impossible entente ? Grands dossiers de Diplomatie, $\mathrm{n}^{\circ}$ 21, p. 74-75.

Bernard J.-P., Gaspard M., Harral C., 1991. L'économie des transports dans l'ex-URSS, Courrier des Pays de l'Est, $\mathrm{n}^{\circ} 363$, p. 3-41.

Besters-Dilgers J., 2005. Le facteur linguistique dans le processus de construction nationale en Ukraine. In Lepesant G. (dir.), L'Ukraine dans la nouvelle Europe, Paris, CNRS-Editions, p. 41-81.

Boyko N., 2005. Églises orthodoxes et identité nationales en Ukraine post soviétique. In Lepesant G. (dir.), L'Ukraine dans la nouvelle Europe, Paris, CNRS-Editions, p. 83-117.

Channon J., 1997. Atlas historique de la Russie, Paris, Autrement, 144 p.

Galtsyan G., 2012. Le renouveau de l'Église orthodoxe russe après la chute de l'URSS. In GuillySulikashvili (dir.), L'énigme russe, pouvoir, économie et société, Septentrion, p. 69-100.

Kalembka W., 1988. Les territoires de l'est dans la pensée politique polonaise, 1837-1870. In Beauvois D. (dir.), Les confins de l'ancienne Pologne, Ukraine, Lituanie, Biélorussie, XVI ${ }^{\circ}-X^{\circ}$ siècle, Presses Universitaires de Lille, p. 145-155. 
Kappeler A., 1994. La Russie, empire multi-ethnique, Paris, Institut d'Études Slaves, 415 p.

Levesque J., 2014. Annexion de la Crimée par la Russie : quelle est la stratégie du Kremlin en Ukraine? Grands dossiers de Diplomatie, ${ }^{\circ} 21$, p. 36-40.

MaksimiukJ., 2006. Transcarpathians Rusyns Want Official Recognition. Rferl.org, 22 septembre.

Marchand P., 2012. Atlas géopolitique de la Russie, la puissance retrouvée. Paris, Autrement, 88 p.

Marchand P., 2014. Géopolitique de la Russie. Paris, PUF, 182 p.

Riasanovski N., 2010. Histoire de la Russie. Paris, Robert Laffont, 843 p.

Rowland R., 2004. National and regional population trends in Ukraine, results from the most recent census. Eurasian Geography and Economics, n 7, p. 491-514.

Ruze A., 1998. De la Moldavie à la Moldova. Les pays de la CEI, édition 1998, La documentation Française, p. 73-89.

Sellier A. et J., 1991. Atlas des peuples d'Europe centrale. Paris, La Découverte, 192 p.

Slekowa L., 1988. L'image des confins dans la littérature. In Beauvois D. (dir.), Les confins de l'ancienne Pologne, Ukraine, Lituanie, Biélorussie, XVIe-XXe siècle, Presses Universitaires de Lille, p. 21-38.

Teurtrie D., 2010. Géopolitique de la Russie. Intégration sociale, enjeux énergétiques, influences culturelles. Paris, L'Harmattan, 348 p.

Thual F., 1993. Géopolitique de l'orthodoxie. Paris, Dunod, 124 p.

\section{NOTES}

1. Ces dates sont celles retenues par Riasanovsky (1987).

2. Le reste de la Bessarabie, annexée par Staline, est devenue la RSS de Moldavie.

3. Pour toute cette partie, les sources sont : Cyclope, US Geological Service, Statistitetchskii Zbirnik «Regioni Ukraiïni ».

4. Selon cet accord, l'Ukraine louait la base jusqu'en 2042, pour un loyer annuel de 100 millions de $\$$ et un rabais de $100 \$$ pour $1000 \mathrm{~m}^{3}$ de gaz (soit en 2013, un rabais de 2,8 milliards de \$).

5. Une partie de ces sources bibliographiques peut se retrouver dans les publications plus volumineuses de l'auteur.

6. Missile balistique à portée intermédiaire.

7. Missile balistique intercontinental.

8. Curieusement, c'est le prix qu'avait obtenu Viktor Ianoukovitch en vertu d'un accord contre lequel l'Ukraine de l'Ouest s'est soulevée et l'a chassé du pouvoir, au motif que son contenu « vendait l'Ukraine à Moscou ».

\section{RÉSUMÉS}

Le territoire de l'Ukraine a été tracé sur la carte en 1945 dans une aire d'affrontement séculaire. Il regroupe des espaces dans lesquels les histoires et les tropismes ne sont pas les mêmes. Mais la 
situation géographique et les liens technologiques d'époque soviétique lient les économies russe et ukrainienne. Plusieurs scénarii se profilent pour l'avenir de la relation Ukraine-Russie, dans lesquels UE et OTAN jouent un rôle important.

Ukrainian territory was drawed on the map in 1945, in an area of immemorial conflicts. This territory is gathering regions with very different history and memory. Geographical situation and soviet technological connections still ties russian en Ukrainian economies. In the future, EU and NATO will play a leading role in relations between the two countries.

\section{INDEX}

Mots-clés : Ukraine, Russie, géopolitique de l'Europe, relations internationales

Keywords : Russia, European geopolitics, international relations

\section{AUTEUR}

\section{PASCAL MARCHAND}

Pascal Marchand, pascal.marchand@hotmail.fr, est Professeur à l'Université de Lyon II, EA 3725 Droit, contrat, territoire, Lyon II. Il a publié récemment :

- Marchand P., Géopolitique de la Russie, PUF, 2014

- Marchand P., Aspect stratégique des axes et des flux : une accélération de la dérive du continent ?, Diplomatie, Grand dossier, $\mathrm{n}^{\circ} 21$, juin-juillet 2014

- Marchand P., Russie et espace eurasiatique, Géoéconomie, septembre 2014. 